\title{
Combination of preoperative neutrophil- lymphocyte ratio, platelet-lymphocyte ratio and monocyte-lymphocyte ratio: a superior prognostic factor of endometrial cancer
}

\author{
Rong Cong ${ }^{1}$, Fanfei Kong ${ }^{1}$, Jian $\mathrm{Ma}^{1}$, Qing $\mathrm{Li}^{1}$, Qijun $\mathrm{Wu}^{2}$ and Xiaoxin $\mathrm{Ma}^{1^{*}}$ (D)
}

\begin{abstract}
Background: The preoperative peripheral blood neutrophil-lymphocyte ratio (NLR), platelet-lymphocyte ratio (PLR) and monocyte-lymphocyte ratio (MLR) have been reported to be associated with the prognosis of various cancers but are always discussed separately. The aim of this study is to bring the combination of NLR, PLR and MLR into the prognostic assessment system of endometrial cancer (EC) and establish a nomogram to provide an objective prediction model for clinical decisions.

Methods: A total of 1111 patients with EC who had accepted surgical treatment during 2013-2017 were involved in the analysis. Their NLR, PLR, and MLR levels were obtained from a routine blood examination within 2 weeks before operation. Receiver operating characteristic curve (ROC) analysis was performed to determine optimal cutoffs. Chi-square tests analysed the associations of the ratios with other clinicopathological variables. The prognostic value was indicated by overall survival (OS) via Cox proportional hazards models and Kaplan-Meier analysis. R software was used to establish the nomogram based on the combination of NLR, PLR, MLR and other clinicopathological factors.

Results: The median follow-up period was 40 months, and the median age was 56 . The enrolled patients were stratified by cutoffs of 2.14 for NLR, 131.82 for PLR and 0.22 for MLR. Multivariate analyses demonstrated that high NLR over $2.14(H R=2.71,95 \% \mathrm{Cl}=1.83-4.02, \mathrm{P}<0.001)$, high $\mathrm{PLR}$ over $131.82(\mathrm{HR}=2.75,95 \% \mathrm{Cl}=1.90-3.97, \mathrm{P}<0.001)$, and high MLR over $0.22(\mathrm{HR}=1.72,95 \% \mathrm{Cl}=1.20-2.45, P=0.003)$ were significantly associated with worse OS. The combined indicator, high NLR + high PLR + high MLR ( $H R=4.34,95 \% \mathrm{Cl}=2.54-7.42, P<0.001)$, showed the highest prognostic value. The Harrell's concordance index of the nomogram was $0.847(95 \% \mathrm{Cl}=0.804-0.890)$, showing good discrimination and calibration of this model.

(Continued on next page)
\end{abstract}

\footnotetext{
* Correspondence: maxiaoxin666@aliyun.com

'Department of Obstetrics and Gynecology, Shengjing Hospital of China

Medical University, Sanhao Street, 110004 Shenyang, People's Republic of

China

Full list of author information is available at the end of the article
}

(C) The Author(s). 2020 Open Access This article is licensed under a Creative Commons Attribution 4.0 International License, which permits use, sharing, adaptation, distribution and reproduction in any medium or format, as long as you give appropriate credit to the original author(s) and the source, provide a link to the Creative Commons licence, and indicate if changes were made. The images or other third party material in this article are included in the article's Creative Commons licence, unless indicated otherwise in a credit line to the material. If material is not included in the article's Creative Commons licence and your intended use is not permitted by statutory regulation or exceeds the permitted use, you will need to obtain permission directly from the copyright holder. To view a copy of this licence, visit http://creativecommons.org/licenses/by/4.0/ The Creative Commons Public Domain Dedication waiver (http://creativecommons.org/publicdomain/zero/1.0/) applies to the data made available in this article, unless otherwise stated in a credit line to the data. 
(Continued from previous page)

Conclusion: The combination of NLR, PLR, and MLR is a superior prognostic factor of EC. The nomogram involving the combination of NLR, PLR, MLR and other clinicopathological factors is recommended to predict OS for EC patients clinically.

Keywords: Endometrial cancer, Monocyte-lymphocyte ratio, Neutrophil-lymphocyte ratio, Platelet-lymphocyte ratio, Prognosis, Nomogram

\section{Background}

Endometrial cancer (EC) is reported as the most common gynaecological tumour with increasing incidence in developed countries [1]. Despite intensive efforts in past decades to improve diagnostic criteria and surgery, radiotherapy and chemotherapy treatments, the management of EC faces the serious risk of recurrence, and cure can be difficult and challenging due to changes in the histological classification, controversy in the usage of lymphadenectomy for early stage patients, decline of adjuvant therapy and discrepant definition of recurrence risk factors based on various classifications [2]. Effective prognostic indicators for early detection of probable recurrence may be helpful for timely and optimal treatment and improve the survival rate. Clinically, noninvasive diagnosis of EC mainly relies on ultrasound and serum cancer antigen 125 (CA-125). However, CA125 may be not a fitting prognostic indicator of EC because it rises in many physiological and pathological conditions, such as menstruation, ovarian cancer, pelvic inflammatory disease and pancreatitis [3]. In Kim's [4] and Ding's [5] studies, the combination of peripheral blood neutrophils and monocytes had higher prognostic value than CA-125.

A simple routine preoperative blood examination is of great importance for predicting the prognosis of patients with EC but is always ignored by clinicians. Since Virchow first noted leukocytes in neoplastic tissues and discussed the relationship between inflammation and cancers in 1881, the peripheral blood neutrophillymphocyte ratio (NLR), platelet-lymphocyte ratio (PLR), and monocyte-lymphocyte ratio (MLR) have been widely used to predict the prognosis of cancers, including gastric cancer, colorectal cancer, lung cancer, oesophageal cancer and breast cancer [6-10]. The indicators from peripheral blood examinations can be collected conveniently for dynamic evaluation of high-risk patients and relieve their financial burden.

However, there is a downside: most existing studies discuss the prognostic value of these ratios separately, and no study focuses on the combination of NLR, PLR, and MLR in EC. In Takahashi's study [11], elevated NLR was not an independent factor of shorter survival for EC patients in multivariate analysis. In Aoyama's study [12], elevated PLR was associated with PFS but not with OS and lymph node metastasis in multivariate analysis. A single ratio is not sufficient nor accurate to evaluate prognosis. Therefore, we integrated the combination of three ratios and some other clinicopathological factors to establish a nomogram model to provide an objective statistical evaluation scale for EC. The nomogram is usually used to provide an individualized evaluation of a certain event by integrating diverse determinant and prognostic variables, and it has been proposed as a better method or even a new standard compared to the traditional staging system in many cases, as reported in several studies $[13,14]$. The aim of the present study is to evaluate the prognostic value of preoperative peripheral blood NLR, PLR, and MLR and their combinations in patients with EC and provide a reliable scoring nomogram model for doctors to make standardized clinical decisions.

\section{Methods \\ Study population}

We retrospectively collected clinicopathological data from patients with EC at our hospital from December 2013 to December 2017. Patients were eligible if they had been diagnosed with primary EC and undergone hysterectomy (with or without adnexectomy and lymphadenectomy). Involved patients were treated according to a detailed preoperative assessment including gynaecological examination, an imaging examination and preoperative histological findings. Patients without full blood count (FBC) data from a time frame of 2 weeks before surgery were excluded. Patients with incomplete clinicopathological data or follow-up information, with atypical hyperplasia or a carcinoid tumour, with other simultaneous malignancies or hematologic disorders, or those that received radiotherapy or chemotherapy before surgery were also excluded.

\section{Data collection}

Clinical information was obtained from archived electronic medical records of the hospital information system. (i) Basic information included the age at surgery, the age at menopause, the body mass index (BMI), reproductive history, complications and smoking history. (ii) Pathological data included the clinical stage, tumour grade, histopathological subtype, depth of myometrial 
tumour invasion, lymphovascular space invasion (LVSI) and surgical method. (iii) Laboratory data included the FBCs (leukocyte, neutrophil, eosinophil, basophil, lymphocyte, monocyte and platelet counts, expressed in $\times 10^{9} / \mathrm{L}$ ). Clinical stage and tumour grade were calculated according to the FIGO 2009 and the histologic typing system of the WHO. The end point index was overall survival (OS), which was defined as the time from the date of the primary surgery to the date of the last follow-up (June 30, 2018) or death from any cause. Death data were obtained from the death certificates.

\section{Statistical analysis}

Statistical analysis was performed using IBM SPSS 19.0 (SPSS, Inc., Chicago, Illinois, USA) and R 3.0.1 software (http://www.Rproject.org). All included patients were stratified according to the preoperative NLR, PLR, and MLR cutoffs, which were generated by a receiver operating characteristic (ROC) curve. The maximum Youden index indicated the optimum cutoff. Survival analysis of the ratios was performed using Kaplan-Meier analysis, and the $P$ value was used to identify significant differences between groups. The Cox proportional hazard models and the Schoenfeld residuals test were conducted to respectively evaluate the hazard ratios and proportional hazard assumptions of the variables. Associations between continuous and categorical variables were analysed using the Mann-Whitney $U$ test and the chi-square test. To test the multicollinearity, we evaluated the variance inflation factors (VIF) and the standard errors. A predictor with VIF $>10$ indicated serious collinearity. A multivariable analysis was conducted on the following covariables: age at surgery $(<55,55-65,65-$ $75, \geq 75$ ), surgical stage (I-IV), tumour grade (1-3), BMI $(<25,25-30, \geq 30)$, diabetes (absent, present), lymphovascular invasion (absent, present) and histopathological subtype (endometrioid, stromal sarcoma, clear cell, serous, mixed, carcinosarcoma). All tests were two-sided, and a $P$ value $<0.05$ was considered statistically significant. R software was used to establish the nomogram to indicate the relationship between possible prognostic factors and actual OS, and Harrell's concordance index (C-index) and a calibration curve were used to measure discriminative capacity. If the $\mathrm{C}$-index is over 0.7 and the calibration curve is approximately matching with the basic curve, the nomogram will be of good prognostic significance.

\section{Results}

In total, 1473 patients with primary EC who underwent hysterectomy between 2013 and 2017 were retrospectively analysed. A total of 345 patients with missing information on any variable were excluded. Another 14 patients who received adjuvant radiotherapy or adjuvant chemotherapy, 2 patients with other simultaneous malignancies and 1 patient with coexistent hematologic disorders were excluded. A total of 1111 patients were included in the analysis. The flow chart of the inclusion and exclusion process is shown in Fig. 1.

The characteristics of the included patients are listed in Table 1. The median age of the enrolled patients was 56 years. The median observation period was 40 months. During observation, a total of 82 patients died. A total of 1008 (90.7\%) patients were diagnosed at stage I or II, while $103(9.3 \%)$ were diagnosed at stage III or IV. The majority of the patients underwent hysterectomy, bilateral salpingo-oophorectomy and lymphadenectomy (78.2\%). The residual tumours were present in 34 patients. The major histopathological subtype was endometrioid endometrial carcinoma (91.9\%).

The median NLR, PLR and MLR were 2.01 (range 0.52-60.44), 121.11 (range 24.06-634.48), and 0.19 (range 0.01-0.83), respectively. ROC curves were generated to identify the optimal cutoffs for the NLR, PLR, and MLR (Fig. 2). A cutoff of 2.14 (area under the curve, AUC $=0.671)$ for the NLR had the highest Youden index. Similarly, cutoffs of $131.82($ AUC $=0.652)$ and 0.22 (AUC $=0.630$ ) were identified for the PLR and MLR, respectively. For further analysis, we dichotomized patients into low and high groups by the cutoffs. Regarding the NLR cutoff, 612 patients (55.1\%) were in the low group, whereas 499 (44.9\%) were in the high group. Regarding the PLR, 647 (58.2\%) patients were in the low group, while $464(41.8 \%)$ were in the high group. Regarding the MLR, 687 (61.8\%) patients had a low MLR, and $424(38.2 \%)$ had a high MLR. Chi-square tests (Table $1, P<0.05$ ) showed that the NLR was significantly associated with patient age and clinical stage, and the PLR was significantly associated with patient age, clinical stage, tumour grade and histopathological subtype. Moreover, significant corrections of the MLR with patient age, clinical stage and tumour grade were revealed.

Table 2 shows that a high NLR, PLR and MLR were associated with poor OS both in the univariate analysis and the multivariate analysis $(P<0.05)$. In the multivariate analysis, the HRs of a high NLR, PLR and MLR were $2.71(95 \% \mathrm{CI}=1.83-4.02, \mathrm{P}<0.001), 2.75$ (95\% CI $=1.90$ 3.97, $\mathrm{P}<0.001)$, and $1.72(95 \% \mathrm{CI}=1.20-2.45, P=0.003)$, respectively. By contrast, the combined indicators, high NLR + high PLR + high MLR, high NLR + high PLR + low MLR, low NLR + high PLR + low MLR, and high NLR + low PLR + high MLR, showed good prognostic value in both the univariate and multivariate analyses. Other combinations were insignificant, as shown in the table. Some clinical characteristics, including patients older than 64 years, patients with Stage III to IV or Grade 2 to 3, patients with lymphovascular space invasion and patients with carcinosarcoma or a mixed 


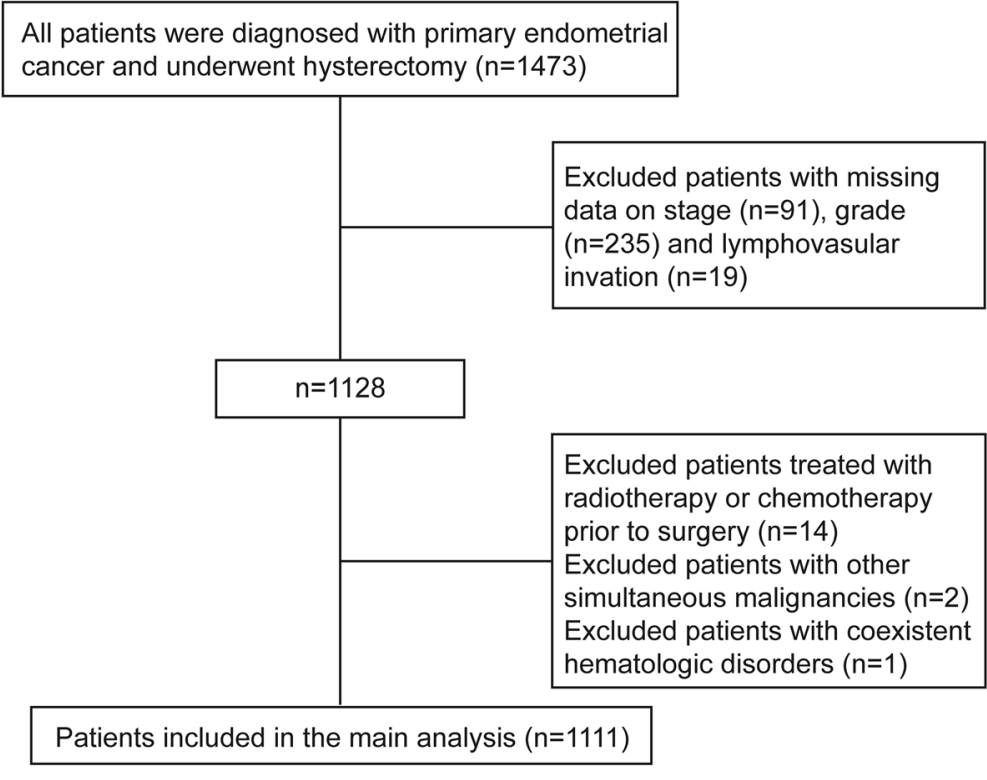

Fig. 1 Flow chart of the included and excluded populations

histopathological subtype, were also significantly associated with OS in both the univariate and multivariate analyses. As the multicollinearity was detected as low level, all the variables were retained in the model. Besides, no violations of proportional hazards or other model assumptions were found by testing Schoenfeld residuals. Kaplan-Meier analysis (Fig. 3) indicated that patients with high preoperative NLR, PLR or MLR had significantly worse OS. The cumulative 5 -year OS rate in the high NLR group was significantly lower than that in the low NLR group (77.9\% vs $92.1 \%$, Fig. 3a). The 5 -year OS rate in the high PLR group was significantly lower than that in the low PLR group $(77.2 \%$ vs $92.0 \%$, Fig. $3 \mathrm{~b})$. The 5 -year OS rate in the high MLR group was significantly lower than that in the low MLR group (82.2\% vs $88.5 \%$, Fig. 3c). For the combined indicators, the 5year OS rate in the high NLR + high PLR + high MLR group was the lowest among all groups (72.4\%, Fig. 3d). The supporting information in Fig. S1 and Fig. S2 showed the combined indicators had stronger connection with lower OS rates both in early stages (Stage I and II) and advanced stages (Stage III and IV).

In the subgroup analysis (full results are provided in supporting information Table S1 and Table S2) of the early (I/II) and advanced (III/IV) stages, the NLR, PLR, and MLR were significantly associated with worse OS in both the early and advanced stages in the univariate analysis. In the multivariate analysis, after adjusting for age, stage, grade, BMI, diabetes, histopathological subtype and lymphovascular space invasion, the NLR, PLR, and MLR were independently prognostic in the early stage $(P<0.05)$, and the PLR and MLR were independently prognostic in the advanced stage $(\mathrm{P}<0.05)$. By contrast, the prognostic values of the combined indicators were consistent in all stages both in the univariate and multivariate analyses. The combined high NLR + high PLR high + high MLR group was associated with the worst OS among all groups (for the multivariate analysis, in early stage, $\mathrm{HR}=4.26,95 \% \mathrm{CI}=2.25-8.08, \mathrm{P}<0.001$; in advanced stage, $\mathrm{HR}=8.91,95 \% \mathrm{CI}=2.97-26.72, \quad \mathrm{P}<$ $0.001)$.

To evaluate the prognostic value of the combined indicators (NLR, PLR and MLR) in EC, a nomogram analysis of OS was performed by the multivariate Cox regression model (Fig. 4a). By summing the points assigned to each variable and drawing a straight line from the total point axis, 1-year probability of survival, 3-year probability of survival and 5-year probability of survival were predicted. The calibration curves showed predicted probability versus actual probability of 3-year and 5-year OS (Fig. 4b-i, ii). The resullt showed an optimal agreement between the prediction using nomogram and the actual observed survival. Furthermore, the Harrell's C-index, the decision curve analysis (DCA), and the AUC of time-dependent ROC curves were used to evaluate the efficacy of the predictive model. The Harrell's C-index of the nomogram was 0.847 ( $95 \% \mathrm{CI}=0.804-0.890)$, but the C-index of the model without the involvement of NLR, PLR and MLR declined to 0.803 (95\% CI $=0.744-$ 0.862). DCA was performed to calculate the clinical net benefit of each model and it was found that the model with the involvement of NLR, PLR and MLR was more benefit than that of the model without NLR, PLR, and MLR in predicting 3-year and 5-year OS (Fig. 4c-i, ii). 
Table 1 Associations of the NLR, PLR and MLR with other clinicopathological variables

\begin{tabular}{|c|c|c|c|c|c|c|c|c|c|c|}
\hline $\begin{array}{l}\text { Clinicopathologic } \\
\text { Characteristics }\end{array}$ & $\begin{array}{l}\text { Total } \\
(\boldsymbol{n}=1111), \\
\text { No (\%) }\end{array}$ & $\begin{array}{l}\text { Low NLR } \\
(\boldsymbol{n}=612 \text {, } \\
55.1 \%) \text {, No (\%) }\end{array}$ & $\begin{array}{l}\text { High NLR } \\
(\boldsymbol{n}=499 \\
44.9 \%), \text { No (\%) }\end{array}$ & $P$ & $\begin{array}{l}\text { Low PLR } \\
(\boldsymbol{n}=647 \text {, } \\
58.2 \%) \text {, No (\%) }\end{array}$ & $\begin{array}{l}\text { High PLR } \\
(\boldsymbol{n}=464 \\
41.8 \%), \text { No (\%) }\end{array}$ & $P$ & $\begin{array}{l}\text { Low MLR } \\
(\boldsymbol{n}=687 \\
61.8 \%), \text { No }(\%)\end{array}$ & $\begin{array}{l}\text { High MLR } \\
(\boldsymbol{n}=424 \\
38.2 \%), \text { No (\%) }\end{array}$ & $P$ \\
\hline \multicolumn{11}{|l|}{ Age, y } \\
\hline$<55$ & $467(42.0)$ & 235 (38.4) & $232(46.5)$ & 0.003 & $223(34.5)$ & $244(52.6)$ & $\begin{array}{l}< \\
0.001\end{array}$ & $276(40.2)$ & $191(45.0)$ & 0.019 \\
\hline $55-64$ & $488(43.9)$ & $298(48.7)$ & $190(38.1)$ & & $326(50.4)$ & $162(34.9)$ & & $324(47.2)$ & $164(38.7)$ & \\
\hline $65-74$ & $131(11.8)$ & 69 (11.3) & $62(12.4)$ & & 85 (13.1) & $46(9.9)$ & & 76 (11.1) & 55 (13.0) & \\
\hline$\geq 75$ & $25(2.3)$ & $10(1.6)$ & $15(3.0)$ & & $13(2.0)$ & $12(2.6)$ & & $11(1.6)$ & $14(3.3)$ & \\
\hline \multicolumn{11}{|l|}{ Stage } \\
\hline 1 & $926(83.3)$ & $523(85.5)$ & 403 (80.8) & 0.027 & $562(86.9)$ & 364 (78.4) & $\begin{array}{l}< \\
0.001\end{array}$ & $592(86.2)$ & 334 (78.8) & 0.003 \\
\hline ॥ & $82(7.4)$ & $43(7.0)$ & $39(7.8)$ & & $39(6.0)$ & $43(9.3)$ & & $44(6.4)$ & $38(9.0)$ & \\
\hline III & $86(7.7)$ & $42(6.9)$ & $44(8.8)$ & & $43(6.6)$ & $43(9.3)$ & & $46(6.7)$ & $40(9.4)$ & \\
\hline IV & $17(1.5)$ & $4(0.7)$ & $13(2.6)$ & & $3(0.5)$ & $14(3.0)$ & & $5(0.7)$ & $12(2.8)$ & \\
\hline \multicolumn{11}{|l|}{ Grade } \\
\hline 1 & $510(45.9)$ & $285(46.6)$ & $225(45.1)$ & 0.19 & $298(46.1)$ & $212(45.7)$ & $\begin{array}{l}< \\
0.001\end{array}$ & $314(45.7)$ & $196(46.2)$ & 0.043 \\
\hline 2 & $378(34.0)$ & 216 (35.3) & $162(32.5)$ & & 246 (38.0) & $132(28.4)$ & & 249 (36.2) & $129(30.4)$ & \\
\hline 3 & $223(20.1)$ & $111(18.1)$ & $112(22.4)$ & & $103(15.9)$ & $120(25.9)$ & & $124(18.1)$ & $99(23.4)$ & \\
\hline \multicolumn{11}{|l|}{$\mathrm{BMI}, \mathrm{kg} / \mathrm{m}^{2}$} \\
\hline$<25$ & $467(42.0)$ & $241(39.4)$ & $226(45.3)$ & 0.096 & $258(39.9)$ & 209 (45.0) & 0.192 & $284(41.3)$ & $183(43.2)$ & 0.406 \\
\hline $25-30$ & $547(49.2)$ & 319 (52.1) & $228(45.7)$ & & 333 (51.5) & $214(33.1)$ & & 337 (49.1) & $210(49.5)$ & \\
\hline$\geq 30$ & $97(8.7)$ & $52(8.5)$ & $45(9.0)$ & & $56(8.7)$ & $41(8.8)$ & & $66(9.6)$ & $31(7.3)$ & \\
\hline \multicolumn{11}{|l|}{ Diabetes } \\
\hline Absent & 918 (82.6) & $498(81.4)$ & $420(84.2)$ & 0.221 & $526(81.3)$ & $392(84.5)$ & 0.167 & $560(81.5)$ & $358(84.4)$ & 0.212 \\
\hline Present & $193(17.4)$ & $114(18.6)$ & 79 (15.8) & & $121(18.7)$ & $72(15.5)$ & & $127(18.5)$ & 66 (15.6) & \\
\hline \multicolumn{11}{|c|}{ Lymphovascular space invasion } \\
\hline Absent & $1081(97.3)$ & $597(97.5)$ & $484(97.0)$ & 0.57 & $631(97.5)$ & $450(97.0)$ & 0.581 & $668(97.2)$ & $413(97.4)$ & 0.864 \\
\hline Present & $30(2.7)$ & $15(2.5)$ & $15(3.0)$ & & $16(2.5)$ & $14(3.0)$ & & $19(2.8)$ & $11(2.6)$ & \\
\hline \multicolumn{11}{|c|}{ Histopathological subtype } \\
\hline Endometrioid & $1021(91.9)$ & $572(93.5)$ & $449(90.0)$ & 0.297 & $610(94.3)$ & $411(88.6)$ & 0.025 & $1021(91.9)$ & $384(90.6)$ & 0.427 \\
\hline $\begin{array}{l}\text { Stromal } \\
\text { sarcoma }\end{array}$ & $4(0.4)$ & $1(0.2)$ & $3(0.6)$ & & $2(0.3)$ & $2(0.4)$ & & $4(0.4)$ & $1(0.2)$ & \\
\hline Clear cell & $6(0.5)$ & $2(0.3)$ & $4(0.8)$ & & $2(0.3)$ & $4(0.9)$ & & $6(0.5)$ & $4(0.9)$ & \\
\hline Serous & $25(2.3)$ & $10(1.6)$ & $15(3.0)$ & & $12(1.9)$ & $13(2.8)$ & & $25(2.3)$ & $13(3.1)$ & \\
\hline Mixed & $25(2.3)$ & $12(2.0)$ & $13(2.6)$ & & $10(1.5)$ & $15(3.2)$ & & $25(2.3)$ & $9(2.1)$ & \\
\hline Carcinosarcoma & $30(2.7)$ & $15(2.5)$ & $15(3.0)$ & & $11(1.7)$ & $19(4.1)$ & & $30(2.7)$ & $13(3.1)$ & \\
\hline
\end{tabular}

NLR neutrophil: lymphocyte ratio; PLR platelet: lymphocyte ratio; MLR monocyte: lymphocyte ratio

The AUC of time-dependent ROC (Fig. 4d) remained in a high place and showed that the nomogram was of good efficacy for predicting prognosis of EC patients with different survival time.

\section{Discussion}

In our study, elevated NLR, PLR and MLR were independent prognostic factors for EC. The findings are consistent with some previous studies $[15,16]$ discussing the prognostic value of the NLR, PLR and MLR for EC, but none of them focused on the combination of the above ratios. Our further combined studies showed that the low NLR + high PLR + high MLR group, the low NLR + low PLR + high MLR group, and the high NLR + low PLR + low MLR group were not significantly associated with OS, indicating that a mere increase in the NLR 

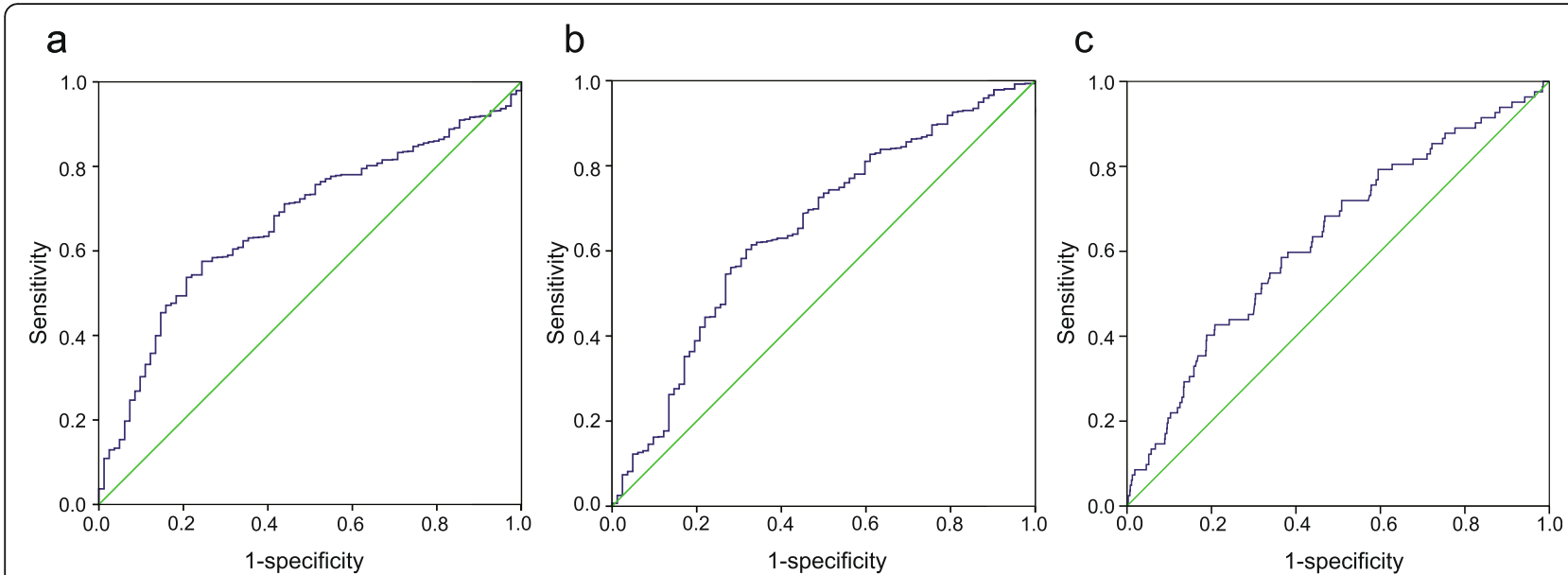

Fig. 2 Receiver operating characteristics (ROC) curve analysis of the NLR, PLR and MLR. a ROC curve analysis of the NLR for OS in endometrial cancer patients. $\mathbf{b}$ ROC curve analysis of the PLR for OS in endometrial cancer patients. c ROC curve analysis of the MLR for OS in endometrial cancer patients

or PLR or MLR without significant changes in the other two indicators would not provide a convincing prognostic value. By contrast, the HRs of a high NLR + a high PLR + a high MLR showed greater prognostic significance and less change in the univariate and multivariate analyses than a single ratio, indicating a more stable prognostic value against other influencing factors.

The present study is, to the best of our knowledge, the first one to discuss the role of the combined preoperative ratios, NLR, PLR, and MLR, in evaluating the prognosis of EC patients. Some previous studies $[11,12]$ had revealed the lack of reliability of the prognostic value of a single ratio in EC. In other cancer models, some scholars $[17,18]$ also doubted the prognostic value of a single ratio and recommended combined ratios as better prognostic indicators. Therefore, it is of great significance to combine the NLR, PLR, and MLR in the evaluation of prognosis to raise diagnostic accuracy.

Besides the three ratios, we also found that some clinical characteristics, including patients older than 64 years, patients with Stage III to IV or Grade 2 to 3, patients with LVSI and patients with carcinosarcoma or a mixed histopathological subtype, were also significantly associated with OS in both the univariate and multivariate analyses. The findings matched the existing studies. Yen's study [19] showed a worse outcome in patients with older age, high-risk histology or high-grade EC after primary surgery. Bendifallah [20] incorporated LVSI into the ESMO classification and achieved better defined indications for EC patients. By contrast, BMI and diabetes did not show a significant association with prognosis in our study. In Mauland's study [21], high BMI is significantly associated with non-aggressive disease in endometrial cancer in the univariate analysis, but not in the multivariate analysis. Binder [22] indicated endometrial cancer was associated with medical comorbidities such as obesity and diabetes, but Mitsuhashi [23] suggested the use of metformin, an antidiabetic drug, could inhibit endometrial cancer cell growth. Therefore the medication usage of EC patients with comorbidities might affect their prognosis and warrant further study. To make the nomogram model more comprehensive, we involved both BMI and diabetes in the assessment system.

The establishment of a nomogram model makes it visible and qualified to evaluate the prognosis with NLR, PLR, MLR and other significant indicators during clinical practice. The model has the advantage of integrating diverse relevant determinants into the prognosis and estimating the individualized risk based on the characteristics of each patient, which usually works better than the subjective judgment of a clinician [24]. In our study, we generated a nomogram model consisting of age, clinical stage, tumour grade, histopathological subtype, lymphovascular space invasion, NLR, PLR and MLR, which could scientifically prognosticate the survival of EC patients by combining blood markers and clinicopathological factors. The calibration curves of 3-year probability of survival and 5-year probability of survival showed that the nomogram had good discrimination and calibration. Ouldamer [25] had reported a nomogram predicting poor prognosis recurrence (PPR) in EC patients, but she did not incorporate NLR, PLR and MLR, and the Harrell's C-index was $0.82(95 \% \mathrm{CI}=0.73-0.89)$. By contrast, the Harrell's C-index was $0.847(95 \% \mathrm{CI}=0.804-0.890)$ in our study, which is also better than similar studies $[26,27]$ focusing on the relationship between cancers and those three ratios. Figure 4a seemed to show that MLR contributed less to the total Nomogram score, but the Harrell's C-index of the nomogram without MLR declined to $0.846(95 \% \mathrm{CI}=0.803-0.889)$, indicating that 
Table 2 Overall survival of the preoperative NLR, PLR and MLR with other clinicopathological variables

\begin{tabular}{|c|c|c|c|c|}
\hline Clinicopathologic Characteristics & Univariate Analysis, HR (95\% Cl) & $P$ & Multivariate Analysis, HR (95\% Cl) & $\mathrm{P}$ \\
\hline \multicolumn{5}{|l|}{ Age, y } \\
\hline$<55$ & 1.00 & & 1.00 & \\
\hline $55-64$ & $0.91(0.62-1.34)$ & 0.622 & $0.99(0.66-1.49)$ & 0.969 \\
\hline $65-74$ & $2.27(1.40-3.67)$ & 0.001 & $2.57(1.56-4.23)$ & $<0.001$ \\
\hline$\geq 75$ & $3.69(1.92-7.11)$ & $<0.001$ & $2.86(1.44-5.68)$ & 0.003 \\
\hline \multicolumn{5}{|c|}{ Stage } \\
\hline 1 & 1.00 & & 1.00 & \\
\hline$\|$ & $2.02(1.17-3.49)$ & 0.012 & $1.34(0.76-2.37)$ & 0.314 \\
\hline III & $6.89(4.69-10.12)$ & $<0.001$ & $3.26(2.13-4.98)$ & $<0.001$ \\
\hline IV & $11.29(6.33-20.14)$ & $<0.001$ & $2.68(1.41-5.10)$ & 0.003 \\
\hline \multicolumn{5}{|l|}{ Grade } \\
\hline 1 & 1.00 & & 1.00 & \\
\hline 2 & $2.43(1.43-4.12)$ & 0.001 & $1.85(1.06-3.23)$ & 0.030 \\
\hline 3 & $8.54(5.21-13.99)$ & $<0.001$ & $3.80(2.22-6.51)$ & $<0.001$ \\
\hline \multicolumn{5}{|l|}{$\mathrm{BMl}, \mathrm{kg} / \mathrm{m}^{2}$} \\
\hline$<25$ & 1.00 & & 1.00 & \\
\hline $25-30$ & $1.21(0.80-1.82)$ & 0.367 & $1.19(0.78-1.82)$ & 0.409 \\
\hline$\geq 30$ & $0.89(0.48-1.66)$ & 0.713 & $1.07(0.57-2.03)$ & 0.828 \\
\hline \multicolumn{5}{|l|}{ Diabetes } \\
\hline Absent & 1.00 & & 1.00 & \\
\hline Present & $1.04(0.65-1.68)$ & 0.854 & $0.94(0.57-1.54)$ & 0.794 \\
\hline \multicolumn{5}{|l|}{ Lymphovascular space invasion } \\
\hline Absent & 1.00 & & 1.00 & \\
\hline Present & $6.75(4.19-10.88)$ & $<0.001$ & $3.11(1.86-5.20)$ & $<0.001$ \\
\hline \multicolumn{5}{|l|}{ Histopathological subtype } \\
\hline Endometrioid & 1.00 & & 1.00 & \\
\hline Stromal sarcoma & $2.34(0.32-16.80)$ & 0.399 & $0.34(0.04-2.83)$ & 0.321 \\
\hline Clear cell & $2.69(0.37-19.35)$ & 0.325 & $0.71(0.09-5.26)$ & 0.733 \\
\hline Serous & $6.91(4.10-11.63)$ & $<0.001$ & $1.02(0.55-1.89)$ & 0.939 \\
\hline Mixed & $4.87(2.45-9.69)$ & $<0.001$ & $2.76(1.31-5.79)$ & 0.007 \\
\hline Carcinosarcoma & $7.76(4.72-12.75)$ & $<0.001$ & $2.80(1.61-4.87)$ & $<0.001$ \\
\hline \multicolumn{5}{|l|}{ NLR } \\
\hline$<2.14$ & 1.00 & & 1.00 & \\
\hline$\geq 2.14$ & $3.96(2.72-5.78)$ & $<0.001$ & $2.71(1.83-4.02)$ & $<0.001$ \\
\hline \multicolumn{5}{|l|}{ PLR } \\
\hline$<131.82$ & 1.00 & & 1.00 & \\
\hline$\geq 131.82$ & $3.54(2.47-5.08)$ & $<0.001$ & $2.75(1.90-3.97)$ & $<0.001$ \\
\hline \multicolumn{5}{|l|}{ MLR } \\
\hline$<0.22$ & 1.00 & & 1.00 & \\
\hline$\geq 0.22$ & $2.12(1.52-2.97)$ & $<0.001$ & $1.72(1.20-2.45)$ & 0.003 \\
\hline \multicolumn{5}{|c|}{ Combined NLR + PLR + MLR } \\
\hline NLR low + PLR low + MLR low & 1.00 & & 1.00 & \\
\hline NLR low + PLR high + MLR high & $1.02(1.57-6.59 \mathrm{E} 157)$ & 0.952 & $<0.001(0.000-5.56 E 197)$ & 0.961 \\
\hline
\end{tabular}


Table 2 Overall survival of the preoperative NLR, PLR and MLR with other clinicopathological variables (Continued)

\begin{tabular}{lllll}
\hline Clinicopathologic Characteristics & Univariate Analysis, HR $(95 \% \mathrm{Cl})$ & $\mathrm{P}$ & Multivariate Analysis, HR (95\% Cl) & $\mathrm{P}$ \\
\hline NLR low + PLR low + MLR high & $0.47(0.10-1.83)$ & 0.252 & $0.40(0.09-1.74)$ & 0.225 \\
NLR high + PLR low + MLR low & $2.07(0.96-4.45)$ & 0.063 & $1.42(0.64-3.15)$ & 0.391 \\
NLR high + PLR low + MLR high & $3.83(1.86-7.89)$ & $<0.001$ & $2.41(1.13-5.15)$ & 0.023 \\
NLR low + PLR high + MLR low & $3.92(2.01-7.62)$ & $<0.001$ & $2.59(1.31-5.11)$ & 0.006 \\
NLR high + PLR high + MLR low & $3.80(1.93-7.47)$ & $<0.001$ & $2.96(1.50-5.89)$ & 0.002 \\
NLR high + PLR high + MLR high & $7.04(4.22-11.76)$ & $<0.001$ & $4.34(2.54-7.42)$ & $<0.001$ \\
\hline
\end{tabular}

Cl, confidence interval; HR, hazard ratio; NLR, neutrophil: lymphocyte ratio; $P L R$, platelet: lymphocyte ratio; $M L R$, monocyte: lymphocyte

the addition of MLR enabled more accurate prognosis prediction. The AUC of time-dependent ROC of the nomogram is also taken to evaluate the efficacy of the nomogram in some studies [28]. The AUC of our model was 0.794 but dropped to 0.777 if MLR was excluded.

Currently, the underlying mechanisms of the associations between the three markers and EC remain poorly understood. Some scholars attribute the association to tumour-induced inflammation and host immunoreaction. Tumour cells could increase peripheral neutrophil levels via stimulating the release of granulocyte colonystimulating factor (GCSF). In turn, neutrophils promote tumour invasion and metastasis via the release of several growth factors such as vascular endothelial growth factor
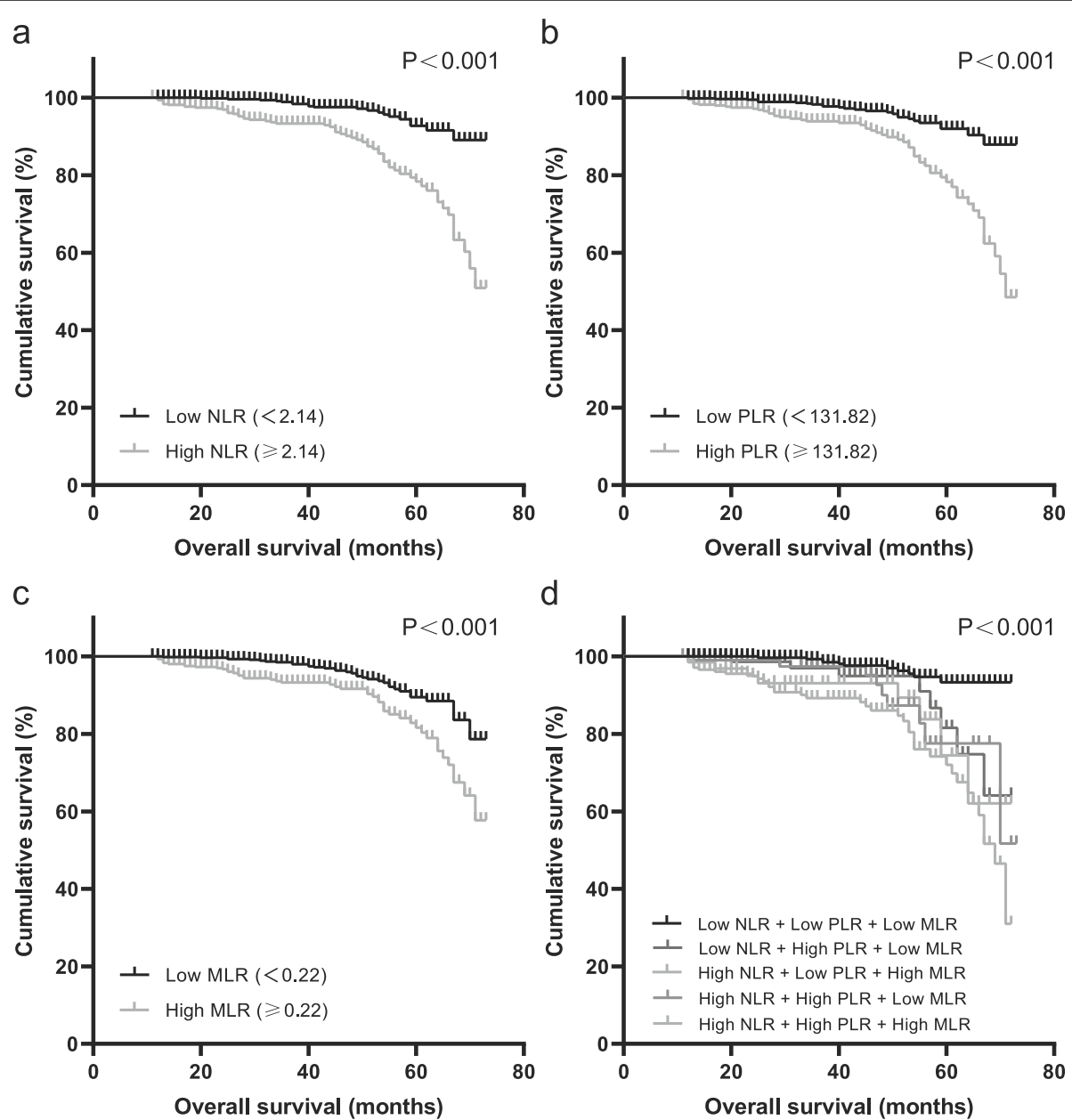

Fig. 3 Overall survival of included patients stratified according to the preoperative NLR, PLR and MLR cutoffs. a Kaplan-Meier curves and $P$ values indicate the relation between OS and the NLR $(P<0.001)$. $\mathbf{b}$ The relation between OS and the PLR $(P<0.001)$. $\mathbf{c}$ The relation between OS and the $\operatorname{MLR}(P<0.001)$. $\mathbf{d}$ The OS comparison of the combined indicators $(P<0.001)$ 


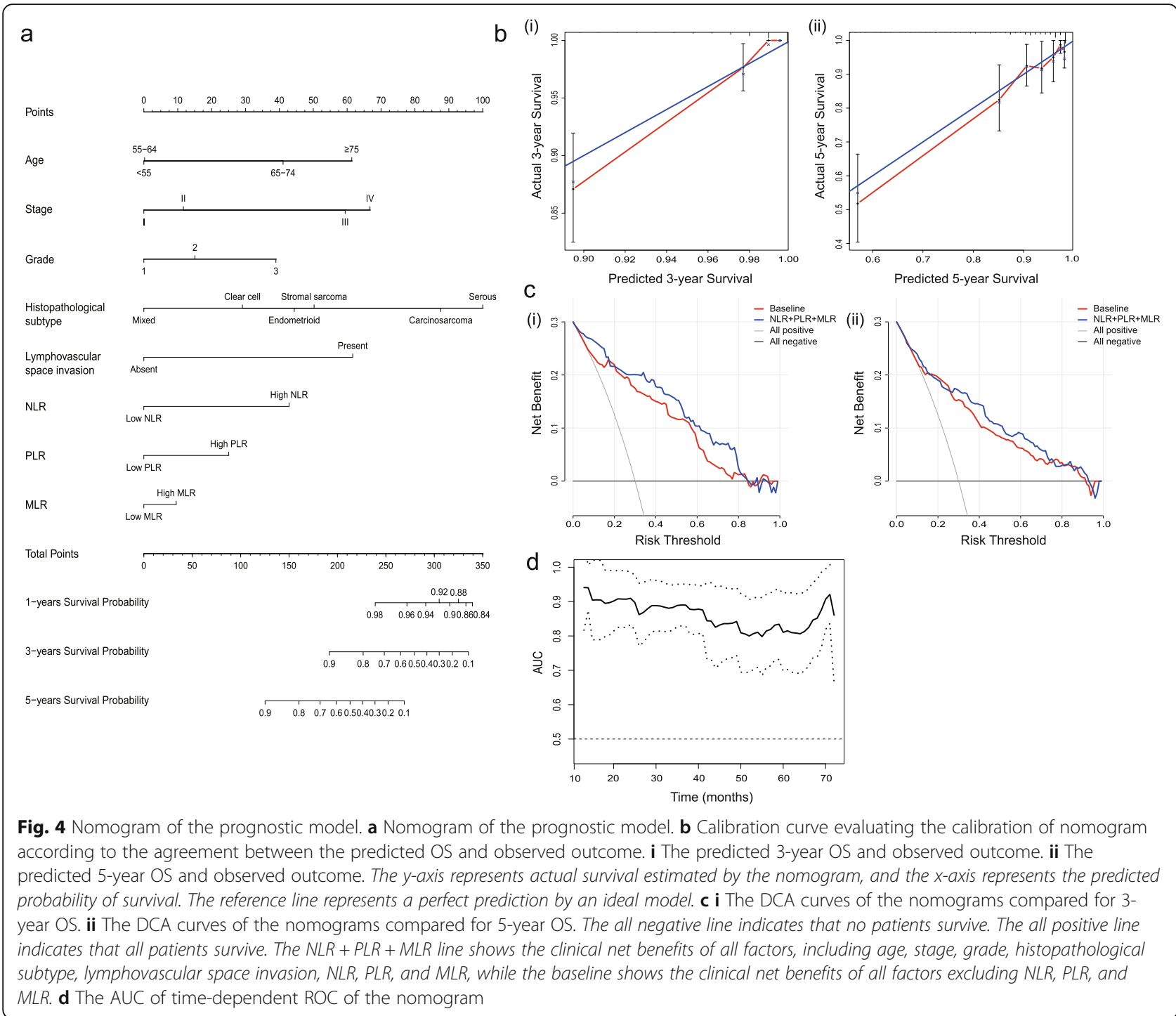

(VEGF) and proteases such as elastases [29]. On the other hand, neutrophils suppress tumour progression via the debridement of hypoxic tumour cells [30]. The hypercoagulable state significantly accounts for a high mortality in cancer patients. Meanwhile, activated platelets release a mass of molecules, such as TXA2, PGE2, $\alpha$-granule contents and exosomes, forming a complex tumour microenvironment that supports the progression of tumour cells. Platelets also aggregate surrounding tumour cells and protect them from elimination by immune cells [31]. Monocytes usually migrate into the tumour microenvironment via VEGF and then differentiate into tumour-associated macrophages (TAMs) induced by tumour chemotactic effects. TAMs produce various factors, such as tumour growth factors and angiogenic factors, to accelerate tumour progression and invasion. On the other hand, monocytes can interfere with the proliferation and activation of lymphocytes, leading to the immune suppression of tumour cells [32]. By contrast, the lymphocyte is usually known as an antitumor effector. In general, CD8 + T lymphocytes play a vital role in attacking tumour cells via cytotoxicity. A lack of CD3 + or CD8 + T lymphocytes in EC was usually believed to be related to poor prognosis [33]. In light of the uncertain mechanisms and some of the bidirectional indicators above, the combination of NLR, PLR and MLR may be a superior prognostic factor of EC that reflects the actual status of tumour-associated immunoreaction.

There are some limitations to our study. First, it was a retrospective study conducted in a single institution, although it is by far the largest study $(n=1111)$ to evaluate the prognostic value of preoperative NLR, PLR and MLR. Secondly, all data on preoperative indicators were collected from patients who later underwent surgical treatment. Therefore, the results might not represent the prognostic value for EC patients with unresectable tumours or other interventions. 


\section{Conclusions}

In summary, the findings of our study indicate that the preoperative blood NLR, PLR, and MLR are independent prognostic markers for OS in EC patients. The combination of NLR, PLR, and MLR provides better prognostic value than any single ratio. The nomogram involving the combination of NLR, PLR, MLR and other clinicopathological factors is recommended as a more convenient and practical model to predict OS for EC patients clinically.

\section{Supplementary information}

Supplementary information accompanies this paper at https://doi.org/10. 1186/s12885-020-06953-8.

Additional file $\mathbf{1}$ Table $\mathbf{S 1}$ Univariate and multivariate analysis of the ratios with other clinicopathological variables in early stages

Additional file $\mathbf{2}$ Table S2 Univariate and multivariate analysis of the ratios with other clinicopathological variables in advanced stages

Additional file $\mathbf{3}$ Figure $\mathbf{S 1}$ Overall survival of patients in early stages stratified according to preoperative NLR, PLR, MLR cut-offs. a KaplanMeier curves and log-rank $P$-values indicated the relation between OS and NLR $(P<0.001)$. b The relation between OS and PLR $(P<0.001)$. c The relation between OS and MLR $(P<0.001)$. d The OS comparison of combined indicators $(P<0.001)$.

Additional file 4 Figure S2 Overall survival of patients in advanced stage stratified according to preoperative NLR, PLR, and MLR cut-offs. a Kaplan-Meier curves and log-rank $P$-values indicated the relation between OS and NLR $(P<0.01)$. b The relation between OS and PLR $(P=0.091)$. c The relation between OS and MLR $(P<0.05)$. d The OS comparison of combined indicators $(P<0.05)$.

\section{Abbreviations}

NLR: Neutrophil-lymphocyte ratio; PLR: Platelet-lymphocyte ratio; MLR: Monocyte-lymphocyte ratio; EC: Endometrial cancer; ROC: Receiver operating characteristic curve; OS: Overall survival; CA-125: Cancer antigen 125; FBC: Full blood count; BMI: Body mass index; LVSI: Lymphovascular space invasion; AUC: Area under the curve; HR: Hazard ratio; Cl: Confidence interval; DCA: Decision curve analysis; PPR: Poor prognosis recurrence; GCSF: Granulocyte colony-stimulating factor; VEGF: Vascular endothelial growth factor; TAMs: Tumour-associated macrophages

\section{Acknowledgements}

We are grateful for the financial support from the National Natural Science Foundation of China, Innovation Team of Higher Education in Liaoning Province, "Major Project Construction Plans" of China Medical University, Distinguished Professor of Liaoning Province and the Outstanding Scientific Fund of Shengjing Hospital.

\section{Authors' contributions}

XM and RC designed and planned the study. RC, FK, JM and $\mathrm{QL}$ collected the data. RC prepared figures and tables and wrote the manuscript. QW helped perform data analysis. All authors have read and approved the manuscript.

\section{Funding}

This work was financially supported by the National Natural Science Foundation of China (No. 81272874 and 81472438) and Innovation Team of Higher Education in Liaoning Province (2018-479) for data collection, "Major Project Construction Plans" of China Medical University (No. 3110118029) for data analysis, Distinguished Professor of Liaoning Province and the Outstanding Scientific Fund of Shengjing Hospital (No. 201601) for writing. The funding body had no involvement in the design of the study, collection, analysis, interpretation of data or in writing the manuscript.

\section{Availability of data and materials}

The data that support the findings of this study are available from the corresponding author upon reasonable request.

\section{Ethics approval and consent to participate}

This study is approved by the Institutional Review Committee of the Ethics Committee of Shengjing Hospital of China Medical University (No.2017PS292K), and it conforms to the provisions of the Declaration of Helsinki.

\section{Consent for publication}

The institutional consent form is available.

\section{Competing interests}

All authors declare that there is no actual or potential conflict of interest.

\section{Author details}

${ }^{1}$ Department of Obstetrics and Gynecology, Shengjing Hospital of China Medical University, Sanhao Street, 110004 Shenyang, People's Republic of China. ${ }^{2}$ Department of Clinical Epidemiology, Shengjing Hospital of China Medical University, Shenyang, People's Republic of China.

Received: 22 January 2020 Accepted: 12 May 2020

Published online: 24 May 2020

References

1. Temur I, Kucukgoz GU, Paydas S, Guzel AB, Sucu M, Vardar MA. Prognostic value of pre-operative neutrophil/lymphocyte ratio, monocyte count, mean platelet volume, and platelet/lymphocyte ratio in endometrial cancer. Eur J Obstet Gynecol Reprod Biol. 2018;226:25-9.

2. Morice $P$, Leary A, Creutzberg C, Abu-Rustum N, Darai E. Endometrial cancer. Lancet. 2016:387:1094-108.

3. Rizner TL. Discovery of biomarkers for endometrial cancer: current status and prospects. Expert Rev Mol Diagn. 2016;6:1315-36.

4. Kim BW, Jeon YE, Cho H, Nam EJ, Kim SW, Kim S, et al. Pre-treatment diagnosis of endometrial cancer through a combination of CA125 and multiplication of neutrophil and monocyte. J Obstet Gynaecol Res. 2012;38: 48-56.

5. Ding L, Ding Y, Mao XH, Zhao JF, Zhou HJ. Retrospective study of the prognostic significance of neutrophil-to-lymphocyte ratio for postsurgical outcomes of patients with endometrial carcinoma. Int J Gynaecol Obstet. 2017;138:311-9.

6. Kijima T, Arigami T, Uchikado $Y$, Uenosono $Y$, Kita $Y$, Owaki T, et al. Combined fibrinogen and neutrophil-lymphocyte ratio as a prognostic marker of advanced esophageal squamous cell carcinoma. Cancer Sci. 2017; 108:193-9.

7. Guo W, Lu X, Liu Q, Zhang T, Li P, Qiao W, et al. Prognostic value of neutrophil-to-lymphocyte ratio and platelet-to-lymphocyte ratio for breast cancer patients: an updated meta-analysis of 17079 individuals. Cancer Med. 2019;8:4135-48

8. Li X, Zeng WH, Zhou YQ, Ji YY, Li WZ, Zhang LY, et al. Neutrophil-tolymphocyte ratio predicted long-term chemotherapy benefits in stage IIIB-IV non-squamous non-small cell lung Cancer patients without sensitive mutations. Onco Targets Ther. 2019;12:8779-87.

9. Dimitriou N, Felekouras E, Karavokyros I, Alexandrou A, Pikoulis E, Griniatsos J. Neutrophils to lymphocytes ratio as a useful prognosticator for stage II colorectal cancer patients. BMC Cancer. 2018;18:1202.

10. Mori M, Shuto K, Kosugi C, Narushima K, Hayashi H, Matsubara H, et al. An increase in the neutrophil-to-lymphocyte ratio during adjuvant chemotherapy indicates a poor prognosis in patients with stage II or III gastric cancer. BMC Cancer. 2018;18:1261.

11. Takahashi R, Mabuchi S, Kawano M, Sasano T, Matsumoto Y, Kuroda H, et al. Prognostic significance of systemic neutrophil and leukocyte alterations in surgically treated endometrial cancer patients: a monoinstitutional study. Gynecol Oncol. 2015;137:112-8.

12. Aoyama T, Takano M, Miyamoto M, Yoshikawa T, Kato K, Sakamoto T, et al. Pretreatment neutrophil-to-lymphocyte ratio was a predictor of lymph node metastasis in endometrial Cancer patients. Oncology. 2019:1-9.

13. Cai YJ, Dong JJ, Dong JZ, Chen Y, Lin Z, Song M, et al. A nomogram for predicting prognostic value of inflammatory response biomarkers in 
decompensated cirrhotic patients without acute-on-chronic liver failure. Aliment Pharmacol Ther. 2017:45:1413-26.

14. Gemenetzis G, Bagante F, Griffin JF, Rezaee N, Javed AA, Manos LL, et al. Neutrophil-to-lymphocyte ratio is a predictive marker for invasive malignancy in Intraductal papillary mucinous neoplasms of the pancreas. Ann Surg. 2017;266:339-45.

15. Eo WK, Kwon S, Koh SB, Kim MJ, Ji Yl, Lee JY, et al. The lymphocytemonocyte ratio predicts patient survival and aggressiveness of endometrial Cancer. J Cancer. 2016;7:538-45.

16. Holub K, Biete A. New pre-treatment eosinophil-related ratios as prognostic biomarkers for survival outcomes in endometrial cancer. BMC Cancer. 2018; 18:1280.

17. Liang Y, Wang W, Li J, Guan Y, Que Y, Xiao W, et al. Combined use of the neutrophil-lymphocyte and platelet-lymphocyte ratios as a prognostic predictor in patients with operable soft tissue sarcoma. J Cancer. 2018:9:2132-9.

18. Huang Z, Liu Y, Yang C, Li X, Pan C, Rao J, et al. Combined neutrophil/ platelet/lymphocyte/differentiation score predicts chemosensitivity in advanced gastric cancer. BMC Cancer. 2018;18:515.

19. Yen MS, Chen TH, Ke YM, Hsu KF, Chen JR, Yu MH, et al. Clinicopathologic Features and Treatment Outcomes in Patients with Stage I, High-Risk Histology or High-Grade Endometrial Cancer after Primary Staging Surgery: A Taiwanese Gynecologic Oncology Group Study. J Clin Med. 2018;7:9.

20. Bendifallah S, Canlorbe G, Raimond E, Hudry D, Coutant C, Graesslin O, et al. A clue towards improving the European Society of Medical Oncology risk group classification in apparent early stage endometrial cancer? Impact of lymphovascular space invasion. Br J Cancer. 2014;110:2640-6.

21. Mauland KK, Trovik J, Wik E, Raeder MB, Njolstad TS, Stefansson IM, et al. High BMl is significantly associated with positive progesterone receptor status and clinico-pathological markers for non-aggressive disease in endometrial cancer. Br J Cancer. 2011;104:921-6.

22. Binder PS, Peipert JF, Kallogjeri D, Brooks RA, Massad LS, Mutch DG, et al. Adult Comorbidity Evaluation 27 score as a predictor of survival in endometrial cancer patients. Am J Obstet Gynecol. 2016;215:766.e1 766.e9.

23. Mitsuhashi A, Kiyokawa T, Sato Y, Shozu M. Effects of metformin on endometrial cancer cell growth in vivo: a preoperative prospective trial. Cancer. 2014;120:2986-95.

24. Balachandran VP, Gonen M, Smith JJ, DeMatteo RP. Nomograms in oncology: more than meets the eye. Lancet Oncol. 2015;16:e173-80

25. Ouldamer L, Bendifallah S, Body G, Touboul C, Graesslin O, Raimond E, et al. Predicting poor prognosis recurrence in women with endometrial cancer: a nomogram developed by the FRANCOGYN study group. Br J Cancer. 2016; 115:1296-303.

26. Wang Y, Sun K, Shen J, Li B, Kuang M, Cao Q, et al. Novel prognostic Nomograms based on inflammation-related markers for patients with hepatocellular carcinoma underwent hepatectomy. Cancer Res Treat. 2019; 51:1464.

27. Choi JH, Suh YS, Choi Y, Han J, Kim TH, Park SH, et al. Comprehensive analysis of the neutrophil-to-lymphocyte ratio for preoperative prognostic prediction Nomogram in gastric Cancer. World J Surg. 2018:42:2530-41.

28. Wang X, Mao M, He Z, Zhang L, Li H, Lin J, He Y, Dai S, Hu W, Liu W. Development and validation of a prognostic Nomogram in AFP-negative hepatocellular carcinoma. Int J Biol Sci. 2019;15(1):221-8.

29. Abu-Shawer O, Abu-Shawer M, Hirmas N, Alhouri A, Massad A, Alsibai B, et al. Hematologic markers of distant metastases and poor prognosis in gynecological cancers. BMC Cancer. 2019;19:141.

30. Blaisdell A, Crequer A, Columbus D, Daikoku T, Mittal K, Dey SK, et al. Neutrophils oppose uterine epithelial carcinogenesis via debridement of hypoxic tumor cells. Cancer Cell. 2015;28:785-99.

31. Takiuchi T, Blake EA, Matsuo K, Sood AK, Brasky TM. Aspirin use and endometrial cancer risk and survival. Gynecol Oncol. 2018;148:222-32.

32. D'Andrea D, Moschini M, Gust KM, Abufaraj M, Ozsoy M, Mathieu R, et al. Lymphocyte-to-monocyte ratio and neutrophil-to-lymphocyte ratio as biomarkers for predicting lymph node metastasis and survival in patients treated with radical cystectomy. J Surg Oncol. 2017;115:455-61.

33. Asaka S, Yen T, Wang T, Shih I, Gaillard S. T cell-inflamed phenotype and increased Foxp3 expression in infiltrating T-cells of mismatch-repair deficient endometrial cancers. Mod Pathol. 2018;32(4):576.

\section{Publisher's Note}

Springer Nature remains neutral with regard to jurisdictional claims in published maps and institutional affiliations.

\section{Ready to submit your research? Choose BMC and benefit from}

- fast, convenient online submission

- thorough peer review by experienced researchers in your field

- rapid publication on acceptance

- support for research data, including large and complex data types

- gold Open Access which fosters wider collaboration and increased citations

- maximum visibility for your research: over $100 \mathrm{M}$ website views per year

At BMC, research is always in progress.

Learn more biomedcentral.com/submissions 